\title{
CONTRIBUIÇÕES DE ATIVIDADES EDUCATIVAS REALIZADAS NA SALA DE ESPERA PARA O ACADÊMICO DE
} ENFERMAGEM

\section{CONTRIBUTIONS OF EDUCATIONAL ACTIVITIES CARRIED OUT IN THE WAITING ROOM FOR THE NURSING ACADEMIC}

\section{CONTRIBUCIONES DE ACTIVIDADES EDUCATIVAS REALIZADAS EN LA SALA DE ESPERA PARA EL ACADÉMICO DE ENFERMERÍA}

Maria Dias Gil ${ }^{1}$, Pâmella Cacciari ${ }^{2}$, Eduardo Fuzzeto Cazañas ${ }^{3}$, Maria Rita Guimarães Maia ${ }^{4}$

\begin{abstract}
RESUMO
Objetivo: relatar a experiência de uma acadêmica de enfermagem no desenvolvimento de atividades na sala de espera e as potencialidades e fragilidades desse processo numa estratégia saúde da família. Método: relato de experiência crítico e reflexivo realizado durante o Estágio Curricular Supervisionado. Resultados: a experiência oportunizou vivenciar algumas fragilidades, sendo elas: dificuldade na elaboração escrita do projeto sobre o tema sugerido, insegurança na abordagem, falta de receptividade de alguns usuários, a intensidade do barulho na sala de espera, que dificultou a comunicação. Além das fragilidades, a acadêmica evidenciou potencialidades, sendo um momento de desenvolver melhor a sua comunicação com o usuário e fortalecer vínculo. Conclusão: A experiência, ao realizar a sala de espera no Estágio Curricular Supervisionado, foi relevante para que a acadêmica pudesse desenvolver estratégias em realizar atividades de educação e promoção de saúde.

Descritores: Enfermagem; Estratégia da saúde da família; Educação em saúde; Capacitação Profissional.
\end{abstract}

\section{ABSTRACT}

Objective: To report the experience of a nursing student in the development of activities in the waiting room and the potentialities and weaknesses of this process in a family health strategy. Method: report of a critical and reflective experience during the Supervised Curricular Internship. Results: the experience allowed observing some weaknesses: difficulty writing the project about the suggested topic, insecurity in the approach, lack of some users' receptivity, the intensity of the noise in the waiting room that hindered communication. In addition to the weaknesses, the academic showed potentialities, being a moment to develop better her communication with the user and strengthen bond. Conclusion: The experience, when performing the waiting room in the Supervised Curricular Internship, was relevant for the academic could develop strategies to carry out activities of health education and promotion.

Descriptors: Nursing; Family health strategy; Health education; Professional Training.

\section{RESUMEN}

Objetivo: Relatar la experiencia de una académica de enfermería en el desarrollo de actividades en la sala de espera y las fortalezas y debilidades de este proceso en una estrategia salud de la familia. Método: relato de experiencia crítica y reflexiva que ocurrió durante el período de Prácticas Curriculares Supervisadas. Resultados: la experiencia proporcionó una oportunidad de experimentar algunos puntos débiles que son: dificultades en la preparación del proyecto de escritura sobre el tema sugerido, la inseguridad en el enfoque, la falta de receptividad de algunos usuarios, la intensidad del ruido en la sala de espera, que dificultó la comunicación. Además de las debilidades, la académica demostró potencialidades, siendo un tiempo para desarrollar mejor su comunicación con el usuario y para fortalecer los lazos. Conclusión: La experiencia de la sala de espera en las Prácticas Curriculares Supervisadas fue relevante para que la académica pudiera desarrollar estrategias en llevar a cabo actividades de educación y promoción de la salud.

Descriptores: Enfermería; Estrategia de salud familiar; Educación en salud; Capacitación Profesional.

${ }^{1}$ Graduada em Enfermagem pela Universidade do Oeste Paulista. ${ }^{2}$ Graduada em Enfermagem. Doutoranda em Gerenciamento em Enfermagem na Universidade Estadual de São Paulo. Docente na Universidade do Oeste Paulista - UNOESTE. ${ }^{3}$ Graduado em Enfermagem. Doutorando em Enfermagem na Universidade Estadual Paulista UNESP. Docente na Universidade do Oeste Paulista - UNOESTE. ${ }^{3}$ Graduanda em Enfermagem pela Universidade Federal de Pelotas. ${ }^{4}$ Graduada em Enfermagem. Doutora em Enfermagem pela Universidade Estadual de Maringá. Docente na Universidade do Oeste Paulista - UNOESTE.

Como citar este artigo:

Gil MD, Cacciari P, Cazañas EF, et al. Contribuições na realização da sala de espera para o acadêmico de Enfermagem. 2018; 8: e2103. Revista de Enfermagem do Centro-Oeste Mineiro. [Access__]; Available in: http://dx.doi.org/10.19175/recom.v7i0.2103 


\section{INTRODUÇÃO}

O modelo de cuidado "Atenção Primária à Saúde" tem sofrido transformações que perpassam assistência às doenças agudas e processos de agudização das doenças crônicas para ações de promoção, prevenção e manejo de condições de saúde ${ }^{(1)}$. Uma das estratégias para essa transição é a Promoção em Saúde (PS), que procura mitigar iniquidade no processo saúde com o objetivo de que o usuário e a comunidade participem ativamente nos fatores que afetam a saúde e qualidade de vida, com o empoderamento do usuário ${ }^{(2)}$.

Um dos cenários em que ocorre a promoção de saúde é a Estratégia de Saúde da Família (ESF), que tem como proposta que os profissionais de saúde não atuem somente no cuidado individual, mas que planejem suas ações direcionadas ao cuidado coletivo. Nessa perspectiva, realizam promoção e prevenção por meio de ações educativas, que também estão inseridas no processo de trabalho dos profissionais de saúde ${ }^{(3)}$.

Na ESF, a sala de espera pode ser utilizada pela equipe em saúde para desenvolver ações de PS, com objetivo de educar os usuários, diminuir o estresse, apoiar, orientar sobre os mitos, amenizar o desgaste emocional e físico da espera por atendimento e detectar problema de saúde. Na maioria dos locais de atendimento à saúde, a sala de espera é um local desagradável, situação em que a espera pelo atendimento que pode gerar angústia, tensão, ansiedade, revolta e comentários negativos sobre o atendimento de saúde ${ }^{(4,5)}$.

A sala de espera é um ambiente de acolhimento, frequentado pelos usuários que utilizam os serviços de saúde. Nessa sala, o profissional da saúde pode proporcionar a humanização do atendimento, como espaços de diálogos e melhora nas relações entre funcionários e usuários, ainda pode ser um apoio para qualidade do atendimento e abordagem ao cuidado integral ao usuário, família e comunidade $^{(5,6)}$.

Portanto, a sala de espera é um local que tem uma grande rotatividade de usuários, com diferentes idades, doenças, cultura e classes sociais. Devido a essa diversidade, a equipe de saúde deve estar preparada para o acolhimento, por meio do diálogo, e avaliar as condições do processo saúde-doença para abordagem do usuário, família e comunidade ${ }^{(7)}$.
Nessa perspectiva, faz-se importante estar ancorado em práticas educacionais colaborativas e compartilhadas entre a gestão, o serviço, academia e população, para que haja diálogo entre todos os envolvidos no processo de saúde, para que não haja sobreposição de saberes, mas sim, integração ${ }^{(8)}$.

As práticas educacionais na sala de espera são relevantes para o vínculo entre a equipe de saúde e usuários, pode proporcionar uma aproximação da comunidade com os serviços de saúde. Esse é um espaço de diálogo e debate que ajuda no estreitamento das relações, sendo um importante alicerce no cuidado humanizado, acolhimento com objetivo de assegurar um olhar holístico ${ }^{(5)}$.

A sala de espera é uma das atividades obrigatórias para os estudantes do Estágio Curricular Supervisionado (ECS) da Atenção Básica, que ocorre no $7^{\circ}$ semestre do curso de enfermagem, com 0 objetivo de realizar promoção em saúde tanto na dimensão individual ou coletiva e ainda proporcionar para o acadêmico, a utilização de diferentes estratégias metodológicas para educação em saúde.

Diante disso, o despertar para o desenvolvimento desse relato de experiência surgiu durante o período em que a acadêmica de enfermagem realizava o ECS. Apesar da contribuição de vários acadêmicos para a atividade da sala de espera, esse trabalho relata a percepção de uma acadêmica que vivenciou todas as etapas desse processo, também possuindo a contribuição de docentes que supervisionam o ECS e as atividades desenvolvidas na sala de espera.

Outro aspecto que instigou os autores a realizar o estudo foi devido à revisão de literatura realizada demonstrar o escasso volume de publicações relacionadas a esse tema.

Dessa forma, a presente investigação tem como objetivo relatar a experiência de uma acadêmica de enfermagem no desenvolvimento de atividades educativas na sala de espera e as potencialidades e fragilidades desse processo na estratégia saúde da família.

\section{MÉTODO}

Estudo descritivo, tipo relato de experiência, desenvolvido a partir de vivências de uma acadêmica de enfermagem no ECS na ESF. Segundo as diretrizes curriculares, o ECS deve ser executado no último ano do curso; ter a 
supervisão do professor e a participação do enfermeiro do serviço de saúde onde se desenvolve o estágio, sendo um procedimento didático para melhor assimilar teoria e prática durante o curso ${ }^{(9)}$.

Este estudo foi desenvolvido numa ESF, no interior do Oeste Paulista, no primeiro semestre de 2016. O município conta com 23 ESF, sendo que o estudo foi realizado numa ESF da região leste, local onde a pesquisadora realizou seu $\mathrm{ECS}^{(10)}$.

A estrutura física da unidade é composta por: recepção, uma sala de triagem, um consultório médico, um consultório odontológico, uma farmácia, uma sala de vacina, uma sala para enfermeira e uma sala de procedimento. A equipe de saúde é composta por uma médica, uma enfermeira, três técnicos de enfermagem, dentista, auxiliar de dentista, oito agentes comunitários de saúde, farmacêutica, técnica de farmácia e uma faxineira. A ESF também tem o apoio do Núcleo de Apoio à Saúde da Família (NASF) com os seguintes profissionais: nutricionista, psicólogo, fonoaudióloga e duas fisioterapeutas residentes.

O estágio que a acadêmica realizou nessa unidade permitiu que a mesma desenvolvesse o projeto "Sala de Espera". A operacionalização das atividades na sala de espera aconteceu em cinco momentos: o primeiro momento ocorreu com a elaboração, pelos acadêmicos de enfermagem, de um projeto para o tema que seria abordado na sala de espera, contendo introdução, objetivo, metodologia, resultados esperados e referências. Após a elaboração do projeto, os acadêmicos enviaram para os docentes, que fizeram adequações necessárias para a realização das atividades na sala de espera. Após o projeto corrigido, o mesmo foi postado num programa da instituição de ensino chamado Aprender, onde todos os acadêmicos tinham acesso.

O segundo momento ocorreu com a organização de como deveria acontecer as atividades na sala de espera, com preparação e estudo de cada tema escolhido pelos estudantes (o qual seria abordado), e a elaboração dos materiais didáticos: cartazes explicativos, ilustrativos e panfletos.
Os temas abordados na sala de espera foram discutidos e escolhidos entre enfermeiros e professores no início do semestre, de acordo com o calendário nacional de saúde. Foi selecionado um tema para cada semana, sendo eles: Prevenção ao câncer de colo de útero e mama; Responsabilidade com o meio ambiente; Dengue; Combate à tuberculose; Promoção à saúde: nutrição e atividade física; Prevenção do câncer; Incentivo à doação de sangue; Prevenção e combate à hipertensão arterial; Dia internacional do trabalhador; Prevenção à dengue: cuidados com a pessoa; Prevenção e cuidados: Zika e o Dia Mundial sem Tabaco.

O terceiro momento se referiu à abordagem dos usuários presentes no ambiente da sala de espera, por meio da explicação dos cartazes disponíveis, da entrega dos panfletos, desenvolvimento dos temas, além do esclarecimento de todas as dúvidas, para melhor compreensão do assunto por todos. As ações na sala de espera ocorreram no período de fevereiro à junho de 2016, no período matutino. O horário proposto para a realização da sala de espera na unidade era das 07h:30 às 08h:30.

No quarto momento, os acadêmicos deveriam lançar as atividades realizadas na sala de espera como produção para a unidade no sistema e-SUS. O quinto momento foi a realização da descrição dos resultados obtidos do projeto "Sala de Espera" pela acadêmica, onde foram discutidos com o enfermeiro e arquivados numa pasta na unidade.

Devido ao fato de este estudo se constituir num relato de experiência, não foi submetido à avaliação de Comitê de Ética. Entretanto, durante seu desenvolvimento, foram considerados os preceitos éticos presentes na Resolução 466/12.

\section{RESULTADOS E DISCUSSÃO}

Ao longo do período do ECS, foram desenvolvidos os diversos temas na Sala de Espera, com diferentes formas de abordagens, com a participação de 129 usuários da unidade, conforme mostra a Figura 1. 
Figura 1- Características da Sala de Espera segundo tema, objetivos, abordagens e número de participantes. Presidente Prudente, SP. 2016.

\begin{tabular}{|c|c|c|c|}
\hline $\begin{array}{c}\text { Temas da Sala de } \\
\text { Espera }\end{array}$ & Objetivos & Abordagens & $\begin{array}{l}\text { Número de } \\
\text { Participantes }\end{array}$ \\
\hline $\begin{array}{l}\text { Prevenção ao câncer do } \\
\text { colo de útero e mama. }\end{array}$ & $\begin{array}{l}\text { Orientar sobre os } \\
\text { métodos prevenção } \\
\text { do Câncer. }\end{array}$ & $\begin{array}{c}\text { Explicação do cartaz com } \\
\text { orientações sobre as formas de } \\
\text { prevenção do câncer. }\end{array}$ & 15 \\
\hline $\begin{array}{l}\text { Prevenção dos diversos } \\
\text { tipos de Câncer }\end{array}$ & $\begin{array}{l}\text { Orientar os usuários } \\
\text { a boas práticas de } \\
\text { saúde para } \\
\text { prevenção de fatores } \\
\text { de risco para } \\
\text { desenvolver câncer. }\end{array}$ & $\begin{array}{c}\text { Entrega de laços nas cores rosa e azul, } \\
\text { simbolizando o câncer de mama e } \\
\text { próstata. Uso de panfletos e cartazes } \\
\text { incentivado mudanças de hábitos } \\
\text { como: prática de exercícios físicos, boa } \\
\text { alimentação, reduzir tabaco, vacina do } \\
\text { HPV, evitar exposição ao sol, entre } \\
\text { outros. }\end{array}$ & 30 \\
\hline $\begin{array}{l}\text { Dia Mundial sem } \\
\text { Tabaco }\end{array}$ & $\begin{array}{l}\text { Orientar os usuários } \\
\text { sobre os riscos que o } \\
\text { tabaco provoca no } \\
\text { corpo. }\end{array}$ & $\begin{array}{c}\text { Orientações para a diminuição do uso } \\
\text { de tabaco com apoio de folders, } \\
\text { cartazes. }\end{array}$ & 07 \\
\hline $\begin{array}{l}\text { Promoção de Saúde: } \\
\text { Nutrição e Atividade } \\
\text { Física. }\end{array}$ & $\begin{array}{l}\text { Orientar sobre os } \\
\text { alimentos saudáveis } \\
\text { e praticar atividade } \\
\text { física. }\end{array}$ & $\begin{array}{l}\text { Entrega de alimentos feitos com } \\
\text { ingredientes saudáveis, p.ex., semente } \\
\text { de chia. }\end{array}$ & 23 \\
\hline $\begin{array}{l}\text { Prevenção e combate à } \\
\text { Hipertensão. }\end{array}$ & $\begin{array}{l}\text { Incentivar os } \\
\text { usuários a mudarem } \\
\text { alguns hábitos } \\
\text { alimentares, p.ex., } \\
\text { diminuir o sal da } \\
\text { comida. }\end{array}$ & $\begin{array}{l}\text { Orientação sobre a hipertensão, } \\
\text { aferição da pressão arterial e anotação } \\
\text { dos resultados numa folha para } \\
\text { controle. }\end{array}$ & 10 \\
\hline $\begin{array}{l}\text { Responsabilidade com o } \\
\text { meio ambiente: } \\
\text { Dengue. }\end{array}$ & $\begin{array}{l}\text { Orientar os usuários } \\
\text { sobre a prevenção da } \\
\text { dengue. }\end{array}$ & $\begin{array}{l}\text { Entrega de panfletos e orientações } \\
\text { sobre sinais e sintomas da dengue, } \\
\text { combate ao mosquito Aedes Aegypti. }\end{array}$ & 25 \\
\hline $\begin{array}{l}\text { Incentivo à Doação de } \\
\text { Sangue. }\end{array}$ & $\begin{array}{l}\text { Explicar como é feito } \\
\text { e incentivar os } \\
\text { usuários a doarem } \\
\text { sangue. }\end{array}$ & $\begin{array}{l}\text { Entrega de panfletos e explicação de } \\
\text { como funciona e os critérios para a } \\
\text { doação de sangue. }\end{array}$ & 26 \\
\hline
\end{tabular}

Fonte: Dados compilados pelos autores.

O Tema prevenção ao câncer do colo de útero e mama ocorreu no dia do mutirão do Papanicolau. Além da coleta de material biológico para o exame, foram realizadas orientações de prevenção do câncer. Orientar as mulheres que aguardam na sala de espera é fundamental, pois o câncer de colo de útero é o terceiro mais frequente nas mulheres, e o de mama é o mais comum nas mulheres do Brasil e do mundo, com maior incidência após os 50 anos. Diversos fatores aumentam os riscos de desenvolver a doença como a idade, hereditariedade e genética, hábitos de vida, história reprodutiva e fatores endócrinos ${ }^{(11)}$.

Quanto ao tema "Prevenção do câncer", foi abordado com "A importância de hábitos de vida saudáveis" e "A realização de exames de rotina".
Alguns tipos de câncer não são unicamente devido a hereditariedade; muitos casos da doença possuem relação com estilo de vida ${ }^{(11)}$. É essencial trabalhar esse tema diante do perfil de mortalidade do Brasil onde as neoplasias são a segunda causa de morte no país ${ }^{(12)}$.

O "Tabaco" foi abordado na sala de espera com o objetivo de sensibilizar o usuário sobre os malefícios para a saúde, inclusive é um fator de risco para desenvolver câncer de pulmão, boca. Segundo a Organização Mundial de Saúde o tabagismo é a principal causa de morte evitável em todo o mundo. Ele é responsável por morte devido a doenças pulmonares crônicas como bronquite e enfisema, cânceres de pulmão, esôfago, laringe, boca, faringe, rim, pâncreas, estomago, fígado, bexiga e colo de útero, e por 
causa de doença coronariana, angina e infarto, e acidente vascular cerebral ${ }^{(13)}$.

O tema "Promoção de saúde: nutrição e atividade física", os usuários foram alertados sobre os benefícios da atividade física e alimentação adequada. De fato, a mudança no estilo de vida impacta na diminuição dos fatores de riscos para as doenças cardiovasculares, sendo a primeira causa de morte no Brasil, responsáveis por cerca de $20 \%$ de todas as mortes na população acima de 30 anos $^{(14)}$.

Outro tema discutido na sala de espera foi a "Prevenção e combate à Hipertensão". Entre as doenças cardiovasculares, a Hipertensão Arterial (HA) é considerada um problema grave de saúde pública. O risco para desenvolver aumenta com a idade, comum em idosos, sendo a doença crônica que mais atinge os idosos que frequentam os serviços de saúde ${ }^{(13)}$.

Diante da prevalência da HA na atenção básica, as orientações sobre o controle adequando da pressão arterial sistêmica, mudança no estilo de vida e a adesão ao tratamento medicamentoso devem ser abordados frequentemente, para que 0 profissional de saúde consiga sanar as dúvidas dos usuários ${ }^{(13)}$.

"A responsabilidade com o meio ambiente: Dengue" foi apresentado na sala de espera no período em que o Brasil estava em epidemia de dengue. Através do diálogo e abordagem com o usuário, percebeu-se uma deficiência de conhecimentos sobre os sinais e sintomas e os locais onde era passíveis a acontecer a proliferação das larvas, uma vez que, nos últimos anos, a dengue transformou-se num problema de saúde pública, devido ao alto número de casos da doença, sendo que a comunidade tem um papel muito importante para poder eliminar o mosquito. Diante dessa situação, o governo tem investido em mais ações na saúde como promoção e prevenção ${ }^{(14,15)}$.

"O incentivo à doação de sangue" deveria ser um tema mais abordado para os usuários nas unidades de saúde, para conscientizar a população sobre esse gesto importante de salvar uma vida. Os hemocentros não conseguem manter uma grande quantidade de sangue, sempre estão precisando de doadores.

A doação de sangue é um problema mundial. Os hemocentros apresentam dificuldades para manter um estoque que atenda aos usuários com emergência, colocando, assim, em risco a saúde e a vida. No Brasil, anualmente, menos de $2 \%$ dos indivíduos realizam a doação de sangue ${ }^{(16,17)}$

Para realizar as atividades na sala de espera, a acadêmica de enfermagem, que participou de toda a operacionalização do processo, encontrou algumas fragilidades para o seu desenvolvimento, sendo elas: dificuldade na elaboração escrita do projeto, insegurança na abordagem aos usuários e a intensidade de barulho no local em que ocorreram as atividades na sala de espera.

Em relação à dificuldade, na elaboração escrita do projeto, sobre os temas sugeridos para a sala de espera, foram identificados pela acadêmica de enfermagem os seguintes motivos: a deficiência na técnica científica, pesquisa em bases de dados, formatação do texto de acordo com as normas da Associação Brasileira de Normas Técnicas (ABNT), a reprodução literal de trechos de autores e parágrafos desconexos.

Existe um número alto de alunos que apresentam dificuldades de escrever e produzir textos. Em enquete realizada com estudantes do curso de Letras da Universidade Federal do Ceará, evidenciou-se que $89 \%$ dos alunos tinham dificuldades para produzir um texto, não conseguindo transmitir informações claras e nítidas para os leitores. Essa preocupação gera angústia e outros fatores psicológicos para os acadêmicos durante a formação ${ }^{(18)}$.

Outro aspecto importante é que, nas Instituições de Ensino Superior, ocorre uma dicotomia entre teoria e prática, ensino e pesquisa, o que reflete na reprodução e não na produção de conhecimentos. Muitos acadêmicos vivenciam a escrita científica apenas no final da graduação pela obrigatoriedade do Trabalho de Conclusão de Curso (TCC). Para suprimir essa deficiência, algumas instituições fomentam a pesquisa através da iniciação cientifica, onde o acadêmico pode fazer pesquisa, adquirir novos saberes e ter uma primeira aproximação com a elaboração de textos com linguagem técnica ${ }^{(19)}$.

"A insegurança na abordagem e falta de receptividade de alguns usuários" também foi uma dificuldade encontrada pela acadêmica de enfermagem, pois atrapalharam no desenvolvimento da comunicação durante a execução do projeto, principalmente no processo de se comunicar com o usuário.

É importante para o acadêmico, o desenvolvimento de habilidades, sendo uma delas a comunicação. A presença do professor supervisor é essencial para dar apoio na interação 
entre o acadêmico e os usuários e com a equipe de saúde, pois há uma resistência no estabelecimento do vínculo, na introdução do acadêmico na equipe de saúde, e a falta de segurança para conversar com os usuários. Essa dificuldade faz parte do desenvolvimento profissional( ${ }^{(20)}$.

Outro aspecto que interferiu na comunicação foram os "Ruídos presentes no ambiente físico da unidade", onde foram realizadas a sala de espera. A literatura descreve a sala de espera como, muitas vezes, um local inadequado, decorrente de falta de espaço, recepção inapropriada e um grande número de pessoas, além do toque do telefone causar ruídos na comunicação ${ }^{(21)}$. Essa intensidade do barulho afetou a execução do projeto da sala de espera, pois os ruídos não permitiam a comunicação de forma efetiva, uma vez que pode impedir que a mensagem emitida seja compreendida pelo receptor, prejudicando a comunicação. Desta forma, no diálogo relacionado entre os profissionais envolvidos e os usuários, deve-se ocorrer uma linguagem clara, pois a mensagem para o usuário pode não ter nenhum significado, ou pode ser compreendido de uma forma errada, não acontecendo a troca de saberes ${ }^{(22)}$.

Muitas vezes, a acadêmica não conseguia desenvolver as atividades propostas na sala de espera devido a outras demandas da unidade, como por exemplo, a coleta de preventivo que ocorria por demanda espontânea e, por esta razão, a acadêmica tinha que parar a atividade para fazer a consulta de saúde da mulher.

Nessa perspectiva, as atividades educativas na sala de espera não são realizadas devido à sobrecarga de trabalho da equipe. Essa mesma percepção foi evidenciada num estudo realizado com 20 enfermeiros de uma ESF do Paraná, em que os mesmos relataram não conseguir empenhar-se nas atividades educativas por motivos de grande demanda de usuários na unidade, de falta de pessoal na equipe, gerenciamento da unidade, trabalhos burocráticos e reuniões, uma vez que essas atividades ocupam um enorme tempo, gerando sobrecarga no serviço ${ }^{(23)}$.

$\mathrm{Na}$ visão da acadêmica de enfermagem, a sala de espera oportuniza a comunicação com o usuário. Essa habilidade é importante para a formação e preparação do acadêmico para a vida profissional futura. Ainda proporciona uma troca de conhecimentos que pode melhorar a insegurança e serve de suporte para que o acadêmico identifique as melhores estratégias de se comunicar com usuário de forma clara e compreensível.

A comunicação é essencial para a realização do processo de promoção de saúde que requer habilidade, competência e atitudes, pois através da comunicação, consegue-se um vínculo com o usuário, confiança e assim, coletar informações sobre seu estado de saúde para poder prestar uma assistência adequada. A formação de vínculo entre equipe, comunidade e família está relacionado entre escuta, humildade e solidariedade entre cada um, havendo o respeito como sujeito e com o desenvolvimento do diálogo ${ }^{(24)}$.

Diante disso, os enfermeiros devem perceber o seu lugar no meio da comunicação, sua importância para garantir uma qualidade da assistência, sendo que ele tem a função de receptor e emissor, que transmite a mensagem de uma forma que o usuário entenda, sendo clara e direta no assunto, para ser compreendida adequadamente, sem que fique confuso ou gere dúvidas. Por meio da comunicação, é possível promover uma qualidade de vida melhor para população ${ }^{(25)}$.

\section{CONCLUSÃO}

Acredita-se que a realização de atividades na sala de espera proporciona ações de educação em saúde que contribuem para uma interação entre a acadêmica de enfermagem e os usuários da ESF, bem como oportuniza a troca de aprendizado para ambos. Estas atividades, além de proporcionarem a troca de experiências, contribuem também para a formação acadêmica.

Em relação aos temas abordados, acreditase que houve uma conscientização da comunidade, pois é através de espaços como estes, que os usuários podem se expressar, opinar, informar-se e refletir sobre os temas propostos, ao mesmo tempo em que esperam pelo atendimento. Permitiu também a sensibilização da equipe sobre a importância de ações como essa para a promoção e prevenção à saúde.

A experiência vivenciada pela acadêmica permitiu detectar algumas fragilidades ocorridas no desenvolvimento de atividades na sala de espera, como dificuldade na elaboração escrita do projeto sala de espera, insegurança na abordagem, a falta de receptividade dos usuários e intensidade do barulho no local onde ocorriam as atividades na sala de espera. 
Realizar atividades educativas na sala de espera possibilitou para a acadêmica um momento para melhorar a sua comunicação com o usuário, compreender a necessidade de realizar promoção da saúde e prevenção de agravos à saúde e colocar em prática políticas públicas. Nessa perspectiva, essa atividade ajudou no desenvolvimento para uma preparação profissional para o futuro.

\section{REFERÊNCIAS}

1. Teixeira MB, Casanova A, Oliveira CCM, Ensgtrom EM, Bodstein RCA. Avaliação das práticas de promoção da saúde: um olhar das equipes participantes do Programa Nacional de Melhoria do Acesso e da Qualidade da Atenção Básica. Saude Debate. 2014;38(spe):52-68. https:// doi.org/10.5935/0103-1104.2014S005

2. Malta DC, Silva MMA, Albuquerque GM, Lima CM, Cavalcante T, Jaime PC, et al. A implementação das prioridades da Política Nacional de Promoção da Saúde, um balanço, 2006 a 2014. Cienc Saude Coletiva. 2014;19(11):4301-12. https:// doi.org/10.1590/1413-

\subsection{4}

3. Brito GEG, Mendes ACG, Santos Neto PM. O objeto de trabalho na Estratégia Saúde da Família. Interface. 2018;22(64):77-86. http://dx.doi.org/10.1590/1807-

$\underline{57622016.0672}$

4. Sato M, Ayres JRCM. Arte e humanização das práticas de saúde em uma Unidade Básica. Interface. 2015:19(55):1027-38. https://doi.org/10.1590/1807-

\subsection{8}

5. Reis FV, Brito JR, Santos JN, Oliveira MG. Educação em saúde na sala de espera: relato de experiência. Rev Med Minas Gerais. 2014;24(Supl

1):S32-S366.

https://doi.org/10.5935/2238-

\subsection{S004}

6. Wild CF, Silveira A, Rosa EO, Favero NB, Rosa EO, Gueterres EC, Leal SDS. Educação em saúde na sala de espera de uma policlínica infantil: relato de experiência. Rev Enferm UFSM. 2014;4(3):660-6. https://
7. Alcântara TV, Shioga JEM, Lima MJV, Lage AMV, Maia AHN. Intervenções psicológicas na sala de espera: estratégias no contexto da oncologia pediátrica. Rev SBPH [Internet]. 2013 [citado 6 fev 2018];16(2):103-19. Disponível em: http://pepsic.bvsalud.org/scielo.php?script= sci arttext\&pid=S1516-08582013000200008 8. Vendruscolo C, Trindade LL, Krauzer IM, Prado ML. A inserção da universidade no quadrilátero da educação permanente em saúde: relato de experiência. Texto Context Enferm. 2016;25(1):e2530013. https:// doi.org/10.1590/0104-07072016002530013

9. Carvalho L, Regebe CMC, Santos ACPO. Professors' reflections on hospital teaching during the supervised academic internship. J Nurs UFPE. 2016;10(7):2731-6. https://doi.org/10.5205/1981-8963v10i7a11334p2731-2736-2016 10. Presidente Prudente (Cidade). Estratégia Saúde da Família - ESF. Presidente Prudente, SP: Secretaria Municipal da Saúde; 2016 [citado 6 nov 2017]. Disponível em: http://www.presidenteprudente.sp.gov.br/sit e/unidades/sms esf.xhtml

11. Prado BBF. Influência dos hábitos de vida no desenvolvimento do câncer. Cienc Cult. 2014 [citado 2018];66(1):21-4. Disponível em: http://cienciaecultura.bvs.br/scielo.php?scrip $\mathrm{t}=\mathrm{sci}$ arttext\&pid=S0009-

67252014000100011

12. Ministério da Saúde (BR). Instituto Nacional de Câncer José Alencar Gomes da Silva (INCA). Estimativa 2018: incidência de câncer no Brasil. Rio de Janeiro, RJ: INCA; 2017.

13. Malta DC, Moura L, Prado RR, Escalante JC, Schmidt MI, Duncan BB. Mortalidade por doenças crônicas não transmissíveis no Brasil e suas regiões, 2000 a 2011. Epidemiol Serv Saude. 2014;23(4):599-608. https://doi.org/10.5123/S167949742014000400002

14. França LS, Vieira SNSV, Lima JJP, Souza FS, Cardim SQ. Health education and the fight against dengue: an experience report. 2017. J Nurs UFPE. 2017; 11(5):2227-30. 
https://doi.org/10.5205/1981-8963-

v11i5a23379p2227-2230-2017

15. Gonçalves RP, Lima EC, Lima JWO, Silva MGC, Caprara A. Contribuições recentes sobre conhecimentos, atitudes e práticas da população brasileira acerca da dengue. Saude Soc. 2015;24(2):578-93.

https://doi.org/10.1590/S0104-

\section{5}

16. Nascimento AA, Ilha $S$, Marzari CK, Diefenbach GD, Backes DS. Cuidado de enfermagem no processo de doação de sangue: percepção dos profissionais e dos doadores. Rev Enferm Cent Oeste Mineiro. 2015;5(1):1497-504.

https://doi.org/10.19175/recom.v0i0.555

17. Dias MS, Prado TO, Santos AHS, Silva FJCP, Santos VS, Lemos LMD. Potenciais doadores de sangue em campanha de sensibilização e captação. Rev Enferm UFPE on 2015;9(2):533-9. https://doi.org/10.5205/1981-8963-

v9i2a10369p533-539-2015

18. Araújo JC, Dieb M. Autoria e deontologia: mediação de princípios éticos e práticas de letramento na escrita acadêmica em um fórum virtual. Rev Bras Lnguist Apl. 2013;13(1):83-104.

https://doi.org/10.1590/S1984-

\section{1}

19. Massi L, Queiroz SL. Estudos sobre iniciação científica no brasil: uma revisão. Cad Pesqui. 2010;40(139):173-97. https://doi.org/10.1590/S0100-

\section{9}

20. Oliveira KRE, Braga EM. O desenvolvimento das habilidades comunicativas e a atuação do professor na perspectiva do aluno de enfermagem. Rev Esc Enferm USP. 2016; 50(esp):32-8. https://doi.org/10.1590/S0080$\underline{623420160000300005}$

21. Dohms M, Tesser CD, Grosseman S. Potencialidades no ensino-aprendizagem da comunicação médico-paciente em três escolas brasileira, espanhola e holandesa. Rev Bras Educ Med. 2013;37(3):311-9. https://doi.org/10.1590/S0100-

\section{$\underline{55022013000300002}$}

22. Broca PV, Ferreira MA. Processo de comunicação na equipe de enfermagem fundamentado no diálogo entre Berlo e King. Esc Anna Nery. 2015;19(3):467-74. https://doi.org/10.5935/14148145.20150062

23. Roecker S, Budó MLD, Marcon SS. Trabalho educativo do enfermeiro na Estratégia Saúde da Família: dificuldades e perspectivas de mudanças. Rev Esc Enferm USP. 2012;46(3):641-9. https://doi.org/10.1590/S00806234201200030001

24. Haddad JGV, Machado EP, Neves-Amado J, Zoboli ELCP. A comunicação terapêutica na relação enfermeiro-usuário da atenção básica: um instrumento para a promoção da saúde e cidadania. Mundo Saude. 2011;35(2):145-55.

https://doi.org/10.15343/0104-

7809.20112145155

25. Bezerra IMP, Machado MFAS, Duarte AS, Costa EAP, Antão JYFL. Comunicação no processo educativo desenvolvido pelos enfermeiros: as tecnologias de saúde em análise. Saude Transform Soc [Internet]. 2014 [citado 6 fev 2018];5(3)42-8. Disponível em: http://www.redalyc.org/articulo.oa?id=2653 35335007

Nota: Este trabalho não possui incentivo de agência de fomento, sendo realizado por recursos próprios dos pesquisadores. Faz parte de Monografia de Final de Curso apresentado para conclusão da graduação em Enfermagem.

Recebido em: 14/06/2017

Aprovado em: 19/02/2018

Endereço de correspondência:

Pâmella Cacciari

Rua Heitor Nogueira de Almeida, 334

CEP: 19067070- - Brasil

E- mail: pamella cacciari@hotmail.com 